\title{
HOMOGENEOUS MANIFOLDS OF ZERO CURVATURE
}

BY

\author{
JOSEPH A. WOLF $\left.{ }^{1}\right)$
}

1. Introduction. It is well known $\left({ }^{2}\right)$ that a connected Riemannian homogeneous manifold of constant curvature zero is isometric to a quotient $R^{n} / D$ where $R^{n}$ is Euclidean space and $D$ is a discrete subgroup of the underlying vector group of $R^{n}$. We will extend that theorem to Lorentz manifolds (this is our main result), to compact manifolds with indefinite metric of arbitrary signature (in contrast to the affine case), and to indefinite-metric manifolds of dimension $<5$. We will then give examples to show that the assumption "compact, or Riemannian, or Lorentz" is essential in dimensions $\mathbf{5}$ or more.

2. Preliminaries. In order to establish terminology and notation, we will recall some definitions. A pseudo-Riemannian manifold $M_{s}^{n}$ is an $n$-dimensional differentiable manifold $M$ with a differentiable family of symmetric bilinear forms $Q_{p}$ on the tangentspaces $M_{p}$ of $M$, where each $Q_{p}$ is equivalent to the form $-\sum_{1}^{s} x_{j} y_{j}+\sum_{s+1}^{n} x_{j} y_{j} . M_{s}^{n}$ has a unique affine connection-the LeviCivita connection on its tangentbundle-with zero torsion and such that parallel translation is a linear isometry (preserves the $Q_{p}$ ) of tangentspaces. $M_{s}^{n}$ is complete if the Levi-Civita connection is complete. $M_{s}^{n}$ is flat, or has constant curvature zero, if the curvature tensor of the Levi-Civita connection is zero. A diffeomorphism of pseudo-Riemannian manifolds is an isometry if it induces linear isometries on the tangentspaces. The group of all isometries of $M_{s}^{n}$ (onto itself) is denoted $\Im\left(M_{s}^{n}\right) ; M_{s}^{n}$ is homogeneous if $\Im\left(M_{s}^{n}\right)$ is transitive on the points of $M_{s}^{n} . M_{s}^{n}$ is complete if it is homogeneous. A pseudoRiemannian covering is a covering $p: N_{s}^{n} \rightarrow M_{s}^{n}$ of connected pseudo-Riemannian manifolds where $p$ is a local isometry; then every deck transformation (homeomorphism $d$ of $N_{s}^{n}$ such that $p=p \cdot d$ ) is an isometry of $N_{s}^{n}$, and, if $N_{s}^{n}$ is simply connected and $D$ is the group of deck transformations of the covering, $M_{s}^{n}$ is homogeneous if and only if the centralizer of $D$ in $\Im\left(N_{s}^{n}\right)$ is transitive on $N_{s}^{n}$ [7, Theorem 2.5]. The holonomy group of $M_{s}^{n}$ at $x$ is the group of linear transformations of the tangentspace $\left(M_{s}^{n}\right)_{x}$ obtained by parallel translation of tangentvectors about sectionally smooth closed curves based at $x$. We will make the convention that a Riemannian manifold is just a pseudo-Riemannian manifold $M^{n}=M_{0}^{n}$ or $M^{n}=M_{n}^{n}$, and a Lorentz manifold is either an $M_{1}^{n}$ or an $M_{n-1}^{n}$.

Received by the editors October 27, 1961.

(1) The author thanks the National Science Foundation for fellowship support during the preparation of this paper.

(2) This is an easy consequence of the work of L. Bieberbach [5]. A somewhat different three-line proof is given in [6]. In addition, we will see that this follows directly from.our Lemma 1. 
The space $V$ of real $n$-tuples, identified with its tangentspace at every point and endowed with the bilinear form $Q(x, y)=-\sum_{1}^{s} x_{j} y_{j}+\sum_{s+1}^{n} x_{j} y_{j}$, carries the structure of a pseudo-Riemannian manifold $R_{s}^{n} . R_{s}^{n}$ is flat because its Levi-Civita connection is the usual affine connection on $V$, and inherits from $V$ the structure of a real vectorspace. Subsets $X$ and $Y$ of $R_{s}^{n}$ are called orthogonal (denoted $X \perp Y$ ) if $Q(X, Y)=0$, and $X^{\perp}$ denotes the largest subspace $W$ of $R_{s}^{n}$ such that $X \perp W$. An element $x \in R_{s}^{n}$ is isotropic if $Q(x, x)=0$, and a subspace $U$ of $R_{s}^{n}$ is totally isotropic if $Q(U, U)=0$. When speaking of $R_{s}^{n}$, we will of ten refer to $Q$, and will usually write $Q(x)$ for $Q(x, x) . \Im\left(R_{s}^{n}\right)$ is the group of all transformations $(A, a): x \rightarrow A x+a$ where $a \in R_{s}^{n}$ and $A$ is a linear transformation of $R_{s}^{n}$ which preserves $Q$; thus $R_{s}^{n}$ is homogeneous. $(A, a)$ is a translation if $A=I$. The usefulness of $R_{s}^{n}$ is due to the fact [7, Theorem 5] that $M_{s}^{n}$ is connected, flat and complete if and only if it admits a pseudoRiemannian covering by $R_{s}^{n}$.

A basis $\left\{v_{i}\right\}$ of $V=R_{s}^{n}$ is called Q-orthonormal if $Q\left(v_{i}, v_{j}\right)=-\delta_{i j}$ for $i \leqq s$ and $Q\left(v_{i}, v_{j}\right)=\delta_{i j}$ for $i>s, \delta_{i j}$ being the Kronecker symbol. Now suppose that $s \leqq n-s$ and that $U$ is a totally isotropic subspace of $R_{s}^{n}$, say $\operatorname{dim}$. $U=l$. One can then find a $Q$-orthonormal basis $\left\{v_{i}\right\}$ of $R_{s}^{n}$ such that, defining $f_{i}=v_{i}$ $-v_{n-l+i}$ and $e_{i}=v_{i}+v_{n-l+i}(1 \leqq i \leqq l),\left\{e_{i}\right\}$ is a basis of $U$ and $\left\{v_{l+1}, \cdots, v_{n-l}\right\}$ $\cup\left\{e_{i}\right\}$ is a basis of $U^{\perp}$. The basis

$$
\left\{f_{1}, \cdots, f_{l} ; v_{l+1}, \cdots, v_{n-l} ; e_{1}, \cdots, e_{l}\right\}
$$

of $R_{s}^{n}$ is called a skew basis with respect to $U$. One has the obvious definition of skew bases for the case $s \geqq n-s$.

Let $p: R_{s}^{n} \rightarrow M_{s}^{n}$ be a pseudo-Riemannian covering, let $D$ be the group of deck transformations, and let $H$ be the holonomy group of $M_{s}^{n}$ at $p(0)$. $p$ gives an identification of $R_{s}^{n}$ with the tangentspace to $M_{s}^{n}$ at $p(0)$, so $H$ may be viewed as acting on $R_{s}^{n}$. This gives us the standard homomorphism $\phi: D \rightarrow H$ by $d=\left(\phi_{d}, t_{d}\right) \in \Im\left(R_{s}^{n}\right) ; \phi$ is onto, and the kernel Ker. $\phi$ is the set of translations in $D$.

If $A$ is a linear transformation, then $\operatorname{Ker} . A$ denotes the kernel and $\operatorname{Im} . A$ the image. If $a$ and $b$ are elements of a group, then $[a, b]$ denotes the commutator $a b a^{-1} b^{-1}$.

3. Computational preparations. Let $D$ be a subgroup of $\Im\left(R_{s}^{n}\right)$, let $Z$ be the centralizer of $D$ in $\Im\left(R_{s}^{n}\right)$, and suppose that $Z$ acts transitively on $R_{s}^{n}$. We adopt the convention that $d, d^{\prime}, d_{i}$ always represent arbitrary elements of $D, d=(R, t)=(I+N, t), d^{\prime}=\left(R^{\prime}, t^{\prime}\right)=\left(I+N^{\prime}, t^{\prime}\right), d_{i}=\left(R_{i}, t_{i}\right)=\left(I+N_{i}, t_{i}\right)$ where $I$ is the identity transformation of $R_{s}^{n}$.

In the proof of Theorem 14.1 of [7], it was seen that transitivity of $Z$ implies:

Lemma 1. If $d \in D$, then $N^{2}=0, t \perp \operatorname{Im} . N$, and $\operatorname{Im} . N$ is totally isotropic.

In the Riemannian case ( $s=0$ or $s=n$ ), there is no nonzero totally isotropic 
subspace of $R_{s}^{n}$, whence the theorem on flat connected Riemannian homogeneous manifolds mentioned in the Introduction.

Lemma 2. If $d \in D$ and $x, y \in R_{s}^{n}$, then $Q(N x, y)+Q(x, N y)=0$, Ker. $N$ $=\operatorname{Im} \cdot N^{\perp}$, Im. $N=\operatorname{Ker} . N^{\perp}$, and $N(t)=0$.

Proof. Lemma 1 gives us $0=Q(R x, R y)-Q(x, y)=Q(N x, y)+Q(x, N y)$ $+Q(N x, N y)=Q(N x, y)+Q(x, N y)$, proving the first statement. Thus $Q(N x, y)=0$ if $N y=0$, so Ker. $N \perp \operatorname{Im} . N$, and the second and third statements follow from the fact that $\operatorname{dim} . \operatorname{Ker} . N+\operatorname{dim} . \operatorname{Im} . N=\operatorname{dim} . \operatorname{Ker} . N+\operatorname{dim} . \operatorname{Ker} . N^{\perp}$ $=\operatorname{dim} . \operatorname{Im} . N+\operatorname{dim} . \operatorname{Im} . N^{\perp}=n$. Finally, $t \in \operatorname{Ker} . N$ because $t \perp \operatorname{Im} . N$ by Lemma 1. q.e.d.

An immediate consequence of Lemmas 1 and 2 is:

Lemma 3. If $d \in D$, then $d^{m}=(I+m N, m t)$ for every integer $m$.

A useful tool for examining commutativity in $D$ is:

Lemma 4. If $d, d^{\prime}, d_{i} \in D$, then $N N^{\prime}+N^{\prime} N=0=N_{1} N_{2} N_{3}$.

Proof. Let $d^{\prime \prime}=d d^{\prime}$; then $N^{\prime \prime}=N+N^{\prime}+N N^{\prime}$, and Lemma 1 gives

$$
0=N^{\prime \prime 2}=N N^{\prime}+N^{\prime} N+N N^{\prime} N+N^{\prime} N N^{\prime}+N N^{\prime} N N^{\prime} \text {. }
$$

Left multiplication of $\left(^{*}\right)$ by $N$ and nonsingularity of $R^{\prime}=I+N^{\prime}$ gives $0=N N^{\prime} N$; right multiplication of $\left({ }^{*}\right)$ by $N^{\prime}$ and nonsingularity of $R=I+N$ gives $0=N^{\prime} N N^{\prime}$. Thus $\left({ }^{*}\right)$ reduces to the first equality of the Lemma. It follows that $0=N_{3}\left(N_{1}+N_{2}+N_{1} N_{2}\right)+\left(N_{1}+N_{2}+N_{1} N_{2}\right) N_{3}=N_{3} N_{1}+N_{1} N_{3}$ $=N_{3} N_{2}+N_{2} N_{3}$, and the second equality follows. q.e.d.

Observe that Lemmas 2 and 4 give us $N^{\prime} N t^{\prime}=-N N^{\prime} t^{\prime}=0$. Thus $d^{\prime \prime}=d d^{\prime}$ and Lemma 2 imply $\left(N+N^{\prime}+N N^{\prime}\right)\left(t+t^{\prime}+N t^{\prime}\right)=0=N t^{\prime}+N^{\prime} t$. It follows that $d d^{\prime} d^{-1}=(I+N, t)\left(I+N^{\prime}, t^{\prime}\right)(I-N,-t)=\left(I+N^{\prime}+2 N N^{\prime}, t^{\prime}+2 N t^{\prime}\right)$ and $\left[d, d^{\prime}\right]=\left(I+2 N N^{\prime}, 2 N t^{\prime}\right)$. This last implies that every translation in $D$ is central, and that the third term in the lower central series of $D$ consists of translations. In summary, we have just proved:

Lemma 5. If $d, d^{\prime} \in D$, then $N^{\prime} N t^{\prime}=0=N N^{\prime} t, N t^{\prime}+N^{\prime} t=0, d d^{\prime} d^{-1}$ $=\left(I+N^{\prime}+2 N N^{\prime}, t^{\prime}+2 N t^{\prime}\right),\left[d, d^{\prime}\right]=\left(I+2 N N^{\prime}, 2 N t^{\prime}\right)$. The set $T=\operatorname{Ker} . \phi$ of all translations in $D$ is central in $D$, and $D$ is nilpotent of order 3.

Here, of course, by nilpotent of order $k$ we mean only that the $(k+1)$ st term of the lower central series is trivial, and do not exclude triviality of the $k$ th term. Observe also that $T$ is the full center of $D$ if the translation parts of elements of $D$ span $R_{s}^{n}$.

4. Commutativity. We retain the notation of $\S 3$, let $\phi: \Im\left(R_{s}^{n}\right) \rightarrow O^{*}(n)$ be the canonical homomorphism, let $H=\phi(D)$, and define $U_{D}$ to be the subspace of $R_{s}^{n}$ spanned by $\{\operatorname{Im} .(\phi(d)-I): d \in D\}$. 
Proposition 1. $R_{s}^{n}$ has a maximal totally isotropic subspace $V_{D}$ which contains $U_{D}$ and on which $H$ acts trivially.

Proof. The Proposition is true for $n=1$, for we are then dealing with a Riemannian signature. Now assume $n>1$ and suppose the Proposition true in dimensions $<n$. We assume $D \neq I$. Then $D$ has a central element $\neq I$ by Lemma 5 , and transitivity of $Z$ shows that the translation part of this element is nonzero. Let $W$ be the subspace of $R_{s}^{n}$ spanned by all translation parts of central elements of $D$; then $W \neq 0$ and Lemma 5 shows that $H$ acts trivially on $W$. We may assume $W^{\perp} \neq 0$, for, if not, then $H=I$ and the Proposition is trivial.

Suppose that $W \cap W^{\perp}=0$. Then $R_{s}^{n}$ is an orthogonal direct sum $W \oplus W^{\perp}$, this decomposition is preserved by $\phi(D)$ and $\phi(Z)$, and $Q$ induces nondegenerate bilinear forms on $W$ and $W^{\perp}$. Thus the Proposition follows by induction on $n$.

Now suppose that $X=W \cap W^{\perp}$ is nonzero. We choose a skew basis

$$
\beta=\left\{f_{1}, \cdots, f_{l} ; v_{l+1}, \cdots, v_{n-l} ; e_{1}, \cdots, e_{l}\right\}
$$

of $R_{s}^{n}$ with respect to $X, l=\operatorname{dim} . X$. As $H$ preserves and acts trivially on $W$, and thus on $X$, every element of $H$ is of the form

$$
h=\left(\begin{array}{ccc}
h_{1}^{\prime} & h_{2}^{\prime} & h_{3}^{\prime} \\
0 & h_{4}^{\prime} & h_{5}^{\prime} \\
0 & 0 & I
\end{array}\right)
$$

in block form relative to $\beta$. The process of restricting to $X^{\perp}$ and passing to $X^{\perp} / X$ shows, by induction on $n$, that the linear span of $\left\{v_{l+1}, \cdots, v_{n-l}\right\}$ has a maximal totally isotropic subspace $Y$ on which each $h_{4}^{\prime}$ acts trivially and which contains the image of each $h_{4}^{\prime}-I$. We define $V_{D}=Y \oplus X . Y \subset X^{\perp}$ shows that $V_{D}$ is totally isotropic, and then it is clear from dimensions that $V_{D}$ is a maximal totally isotropic subspace of $R_{s}^{n}$ which is $H$-invariant. Let $\beta^{\prime}$ be a skew basis of $R_{s}^{n}$ with respect to $V_{D}$. Every $h \in H$ has form

$$
h=\left(\begin{array}{lll}
h_{1} & h_{2} & h_{3} \\
0 & h_{4} & h_{5} \\
0 & 0 & h_{6}
\end{array}\right)
$$

relative to $\beta^{\prime}=\left\{f_{1}^{\prime}, \cdots, f_{m}^{\prime} ; v_{m+1}^{\prime}, \cdots, v_{n-m}^{\prime} ; e_{1}^{\prime}, \cdots, e_{m}^{\prime}\right\} . h_{2}=0$ because (Lemma 1) $\operatorname{Im} .(h-I)$ is totally isotropic and the linear span $S$ of $\left\{v_{m+1}^{\prime}, \cdots, v_{n-m}^{\prime}\right\}$ is a positive or negative definite subspace; it follows that $h_{5}=0$ because $h \in O^{*}(n)$. Similarly, $h_{4}=I$. We wish to prove $h_{1}=I$; then $h \in O^{*}(n)$ will imply $h_{b}=I$, we will have 


$$
h=\left(\begin{array}{lll}
I & 0 & h_{3} \\
0 & I & 0 \\
0 & 0 & I
\end{array}\right)
$$

relative to $\beta^{\prime}$, and the Proposition will be proved. To prove $h_{1}=I$, it suffices to prove $\operatorname{Im}$. $(h-I) \subset V_{D}$. As $\operatorname{Im}$. $(h-I)$ is totally isotropic and $V_{D}$ is maximal totally isotropic, it suffices to show $\operatorname{Im}$. $(h-I) \perp V_{D}$. It is clear that $\operatorname{Im}$. $(h-I)$ $\perp Y$, so we need only show $\operatorname{Im}$. $(h-I) \perp X$. Looking at $h$ in the basis $\beta$, this is clear because $h_{1}^{\prime}=I$. q.e.d.

Proposition 2. Let $\beta$ be a skew basis of $R_{s}^{n}$ with respect to $U_{D}$. Then every $h \in H$ is of the form

$$
h=\left(\begin{array}{ccc}
I & 0 & \alpha_{h} \\
0 & I & 0 \\
0 & 0 & I
\end{array}\right)
$$

relative to $\beta$, where $\alpha_{h}$ is skew-symmetric; $H$ has an element $h_{0}$ such that $\alpha_{h_{0}}$ is nonsingular; the translation part of every element of $D$ lies in $U_{D}^{\perp}$, and $D$ is represented faithfully as a group of translations of $U_{D}^{\perp}$. In particular, $D$ and $H$ are torsion-free abelian groups.

Proof. The form of the elements $h \in H$ relative to $\beta$ is clear from Proposition 1. We will write $\alpha_{i}$ for $\alpha_{h_{i}}$. If $h_{1}=h_{2} h_{3}$, then $\alpha_{1}=\alpha_{2}+\alpha_{3}$; the existence of $h_{0}$ is now clear. Let $d_{0}=\left(h_{0}, t_{0}\right) \in D$. Then $t_{0} \in \operatorname{Ker} .\left(h_{0}-I\right)=U_{D}^{\perp}$ by Lemmas 1 and 2 and nonsingularity of $\alpha_{0}$. Now let $d_{1}=\left(h_{1}, t_{1}\right)$ be any element of $D$, and observe that one can find integers $u$ and $v$ such that $u \alpha_{0}+v \alpha_{1}$ is nonsingular. Let $d_{2}=d_{1}^{0} d_{0}^{u}=\left(h_{2}, t_{2}\right)$; then $\alpha_{2}=u \alpha_{0}+v \alpha_{1}$ is nonsingular and $t_{2}=v t_{1}+u t_{0} \in U_{D}^{\perp}$. Thus $t_{1} \in U_{\bar{D}}^{\perp}$. The Proposition follows. q.e.d.

5. The main results. Our main result is the Lorentz case of the following Theorem 1. As mentioned in the Introduction, the Riemannian case is known from the work of L. Bieberbach [5]. The compact case is interesting in relation of the work of L. Auslander on compact locally affine spaces $([1 ; 2 ; 3$ and 4], for example), and shows that the metric plays a strong role.

TheOREM 1. Let $M_{8}^{n}$ be a connected flat homogeneous pseudo-Riemannian manifold, and suppose that

1. $M_{s}^{n}$ is compact; or

2. $M_{s}^{n}$ is Riemannian, i.e., $s=0$ or $s=n$; or

3. $M_{s}^{n}$ is Lorentzian, i.e., $s=1$ or $s=n-1$; or

4. the dimension $n=\operatorname{dim} . M_{s}^{n} \leqq 4$.

Then $M_{s}^{n}$ is isometric to a quotient $R_{s}^{n} / D$ where $D$ is a discrete group of translations of $R_{s}^{n}$. This result is best possible in the sense that, if $n>4$ and if $s \neq 0,1$, $n-1$ or $n$, then there is a connected noncompact flat homogeneous $N_{s}^{n}$ with nontrivial holonomy group. 
Proof. Let $D$ be the group of deck transformations of the universal pseudo-Riemannian covering $\pi: R_{s}^{n} \rightarrow M_{s}^{n}$, let $Z$ be the centralizer of $D$ in $\Im\left(R_{s}^{n}\right)$, and let $H$ be the holonomy group of $M_{s}^{n}$ at $\pi(0) . Z$ is transitive on $R_{s}^{n}$ by homogeneity of $M_{s}^{n}$; this allows us to use the results of $\S 4$.

We adopt the terminology of $\S 4$. If $M_{s}^{n}$ is compact, then the translation parts of the elements of $D$ span $R_{s}^{n}$, whence $U_{D}^{\perp}=R_{s}^{n}$ by Proposition 2 ; thus $U_{D}=0$. If $M_{s}^{n}$ is Riemannian or Lorentzian, then every totally isotropic subspace of $R_{s}^{n}$ has dimension $<2$; thus $m=\operatorname{dim} . U_{D}<2$. If $m \neq 0$, then Proposition 2 gives us a skew nonsingular $m \times m$ matrix $\alpha_{h_{0}}$; it follows that $m=0$; thus $U_{D}=0$. If $n \leqq 4$ and $M_{s}^{n}$ is neither Riemannian nor Lorentzian, then $s=2$ and $n=4$. Then, if $U_{D} \neq 0$, Proposition 2 would give us $d=(h, t) \in D$ with $0 \neq t$ $\in U_{D}=\operatorname{Im} .(h-I)$. This gives $v \in R_{s}^{n}$ with $t=(h-I) v$, whence $d(-v)=h(-v)$ $+(h-I) v=-v$, contradicting the fact that $d$ has no fixed point.

Now $U_{D}=0$ in all four cases, whence $H=I$ and $D$ consists of translations. $D$ is discrete because $M_{s}^{n}=R_{s}^{n} / D$ is a manifold.

The manifolds $N_{s}^{n}$ will be constructed in $\$ 6$, completing the proof of Theorem 1 , in such a way as to show the bounds of Theorem 2 to be best possible.

THEOREM 2. Let $M_{s}^{n}$ be a connected flat homogeneous pseudo-Riemannian manifold, let $D$ be the group of deck transformations of the universal pseudoRiemannian covering $\pi: R_{s}^{n} \rightarrow M_{s}^{n}$, let $H$ be the holonomy group of $M_{s}^{n}$, and let $\phi: D \rightarrow H$ be the standard homomorphism. Then $D$ is free abelian on some number $m \leqq n$ of generators, $D$ is represented faithfully as a discrete group of translations of a linear subspace of $R_{s}^{n}, D$ has a subgroup $D^{\prime}$ such that $D=D^{\prime} \times \operatorname{Ker} . \phi$, and $m \leqq n-2$ in case $H \neq I . M_{s}^{n}$ has the homotopy type of an $m$-torus; its EulerPoincaré characteristic is zero, and its integral cohomology is an exterior algebra on $m$ generators $\left({ }^{3}\right)$.

Proof. The first part follows easily from Proposition 2, in the same manner as the proof of the first part of Theorem 1 . To prove the second part, we observe that both $M_{s}^{n}$ and an $m$-torus are Eilenberg-MacLane spaces $K(D, 1)$.

6. An example. In order to complete the proof of Theorem 1 and show the bound of Theorem 2 to be best possible, we take integers $n$ and $s$ with $2 \leqq s \leqq n-2$ and $n>4$, and will construct connected flat homogeneous pseudoRiemannian manifolds $N_{s}^{n}=R_{s}^{n} / D$ with nontrivial holonomy group and with $D$ free abelian on $m=n-2$ generators.

Let $\left\{v_{i}\right\}$ be an "orthonormal" basis of $R_{s}^{n}$, and let $U$ be a two-dimensional totally isotropic subspace of $R_{s}^{n}$ such that we have a skew basis $\beta=\left\{f_{1}, f_{2} ; v_{3}, \cdots, v_{n-2} ; e_{1}, e_{2}\right\}$ of $R_{s}^{n}$ with $f_{i}=v_{i}+v_{n-2+i}, e_{i}=v_{i}-v_{n-2+i}$, and $\left\{e_{1}, e_{2}\right\}$ is a basis of $U$. Let

(3) Compare with $[2 ; 3 ; 4]$, and the proof of $[1$, Lemma 1$]$. 


$$
h=\left(\begin{array}{lll}
I & 0 & J \\
0 & I & 0 \\
0 & 0 & I
\end{array}\right)
$$

relative to $\beta$, where

$$
J=\left(\begin{array}{rr}
0 & 1 \\
-1 & 0
\end{array}\right)
$$

and observe that $h$ is in the orthogonal group of $R_{s}^{n}$. We now define elements $d_{1}, d_{2}, \cdots, d_{n-2}$ of $\Im\left(R_{s}^{n}\right)$ by $d_{1}(x)=h(x)+v_{3}, d_{2}(x)=x+v_{4}, \cdots, d_{n-4}(x)$ $=x+v_{n-2}, d_{n-3}(x)=x+e_{1}$, and $d_{n-2}(x)=x+e_{2}$. Let $D$ be the subgroup of $\Im\left(R_{s}^{n}\right)$ generated by the $d_{i}$, and let $Z$ be the centralizer of $D$ in $\Im\left(R_{s}^{n}\right)$. It is clear that $D$ is free abelian on $n-2$ generators, that $N_{s}^{n}=R_{s}^{n} / D$ is a manifold, and that $N_{s}^{n}$ has nontrivial holonomy group generated by $h$. Thus we need only prove that $N_{s}^{n}$ is homogeneous, i.e., that $Z$ is transitive on the points of $R_{p}^{n}$.

To prove transitivity of $Z$, let $v$ be an arbitrary element of $R_{s}^{n}$; we must find $(z, v) \in \Im\left(R_{s}^{n}\right)$ which commutes with each $d_{i}$. Write $v=a_{1} f_{1}+a_{2} f_{2}{ }^{\perp} w$ where $w \in U^{\perp}$. To construct $z$, we define a linear transformation $z^{\prime}$ of $U^{1}$ by $v_{3} \rightarrow v_{3}-a_{2} e_{1}+a_{1} e_{2}, v_{i} \rightarrow v_{i}$ for $3<i \leqq n-2$, and $e_{j} \rightarrow e_{j}$. This preserves the bilinear form induced on $U^{\perp}$ by $Q$, and thus extends to an element of the orthogonal group $O^{s}(n)$ of $R_{s}^{n}$, say $z$, by Witt's Theorem. As $z\left(v_{i}\right)=v_{i}$ for $3<i \leqq n-2$ and $z\left(e_{j}\right)=e_{j}$, it follows that $(z, v)$ commutes with $d_{k}$ for $k>1$. To see that $(z, v)$ commutes with $d_{1}$, we must show that $z h=h z$ and $(z-I) v_{3}$ $=(h-I) v$. The second condition is satisfied by construction of $z^{\prime}$. Now

$$
z=\left(\begin{array}{lll}
z_{1} & z_{2} & z_{3} \\
0 & z_{4} & z_{5} \\
0 & 0 & z_{6}
\end{array}\right)
$$

in block form relative to $\beta$. By construction of $z^{\prime}, z_{4}=I$ and $z_{6}=I$. Then $z \in O^{*}(n)$ gives $z_{1}=I$. Thus

$$
z h=\left(\begin{array}{ccc}
I & z_{2} & J+z_{3} \\
0 & I & z_{5} \\
0 & 0 & I
\end{array}\right)=h z
$$

in block form relative to $\beta$, proving transitivity of $Z$ on $R_{s}^{n}$.

\section{REFERENCES}

1. L. Auslander, On the group of affinities of locally affine spaces, Proc. Amer. Math. Soc. 9 (1958), 471-473. 
2. - On the Euler characteristic of compact locally affine spaces, Comment. Math. Helv. 35 (1961), 25-29.

3. - On the Euler characteristic of compact locally affine spaces. II, Bull. Amer. Math. Soc. 67 (1961), 405-406.

4. - On radicals of discrete subgroups of Lie groups, with applications to locally affine spaces, to appear.

5. L. Bieberbach, Über die Bewegungsgruppen der Euklidischen Raüme, Math. Ann. 70 (1911), 297-336; II, Math. Ann. 72 (1912), 400-412.

6. J. A. Wolf, Sur la classification des varietes riemanniennes homogènes d courbure constante, C. R. Acad. Sci. Paris 250 (1960), 3443-3445.

7. - Homogeneous manifolds of constant curvature, Comment. Math. Helv. 36 (1961), 112-147.

The Institute for Advanced Study,

Princeton, New Jersey 